\title{
ISOMORPHISM CONJECTURE FOR BAUMSLAG-SOLITAR GROUPS
}

\author{
F. THOMAS FARRELL AND XIAOLEI WU
}

Aвstract. In this paper, we prove the K- and L-theoretical Isomorphism Conjecture for Baumslag-Solitar groups with coefficients in an additive category.

\section{INTRODUCTION}

In this paper, we confirm that the Isomorphism conjecture is true for all BaumslagSolitar groups. Our main theorem is as follows

Theorem 0.1. The K- and L-theoretical Isomorphism Conjecture is true for every BaumslagSolitar group with coefficients in any additive category.

Remark 0.2. Independently, G. Gandini, S. Meinert and H. Rüping proved the Isomorphism Conjecture with coefficients in an additive category for the fundamental group of any graph of abelian groups in [5], which includes all Baumslag-Solitar groups.

Recall that the Baumslag-Solitar group $B S(m, n)$ is defined by $\left\langle a, b \mid b^{-1} a^{m} b=a^{n}\right\rangle$ and all the solvable ones are isomorphic to $B S(1, n)$. Note that $B S(m, n) \cong B S(n, m) \cong$ $B S(-m,-n)$. When $m= \pm n, B S(m, \pm m)$ are CAT(0) groups, hence one can use [1] and [8] to conclude $B S(m, \pm m)$ satisfies the Isomorphism Conjecture with coefficients in an additive category. Since the construction of a $K(B S(m, \pm m), 1)$ space with universal cover a CAT(0) space is enlightening for part of our proof, we construct it here. We start with two cylinders $S_{1}^{1} \times[0,1], S_{2}^{1} \times[0,1]$ with $S_{1}^{1}$ is the standard circle with radius $m, S_{2}^{1}$ has radius 1 . We will glue $S_{1}^{1} \times\{0\}$ to $S_{2}^{1} \times\{0\}$ by a $m$-fold (orientation preserving) local isometry covering map. And we do the same to $S_{1}^{1} \times\{1\}$ and $S_{2}^{1} \times\{1\}$. What we get is a $K(B S(m, m), 1)$ space with universal cover CAT $(0)$. If we reverse orientation on the second gluing, we will get a $K(B S(m,-m), 1)$ space with universal cover CAT(0).

In this paper, we will actually prove the Isomorphism conjecture for $B S(m, n)$ with finite wreath products and coefficients in an additive category. The K- and L-theoretical Isomorphism Conjecture with finite wreath products and coefficients in an additive category is known for all solvable Baumslag-Solitar groups, see [6] and [8]. Hence we only need to

Date: September 22, 2013 and, in revised form, April 1, 2014.

2010 Mathematics Subject Classification. 18F25,19A31,19B28.

Key words and phrases. Isomorphism Conjecture; K-theory of group rings; L-theory of group rings; BassSerre theory.

The first author was supported in part by NSF Grant DMS 1206622. 
prove the case when $m>|n|>1$. We will abbreviate the K- and L-theoretical Isomorphism Conjecture with finite wreath products and coefficients in an additive category by FJCW. The idea is to analyze the preimages of cyclic subgroups corresponding to a certain linear representation of $B S(m, n)$ by using Bass-Serre theory, and conclude they satisfy FJCw. Our results relies on previous work on $\mathrm{FJCw}$ for $\mathrm{CAT}(0)$ groups and virtually solvable groups by Bartels and Lück in [1], and Wegner in [7], [8]. Note that FJCw implies the Isomorphism Conjecture with coefficients in an additive category.

\section{Inheritance Properties and Results on FJCw}

We list some inheritance properties and results on $\mathrm{FJCw}$ that we will need. For more information about $\mathrm{FJCw}$ we refer to [8] section 2.3.

Proposition 1.1. (1) If a group $G$ satisfies $F J C w$, then every subgroup $H<G$ satisfies FJCw.

(2) If $G_{1}$ and $G_{2}$ satisfy $F J C w$, then their direct product $G_{1} \times G_{2}$ and free product $G_{1} * G_{2}$ satisfy $F J C w$.

(3) Let $\left\{G_{i} \mid i \in I\right\}$ be a directed system of groups (with not necessarily injective structure maps). If each $G_{i}$ satisfies FJCw, then the direct limit colim ${ }_{i \in I} G_{i}$ satisfies FJCw.

(4) Let $\phi: G \rightarrow Q$ be a group homomorphism. If $Q$ and $\phi^{-1}(C)$ satisfy FJCw for every cyclic subgroup $C<Q$ then $G$ satisfies FJCw.

(5) CAT(0) groups satisfy FJCw.

(6) Virtually solvable groups satisfy FJCw. In particular $\mathbb{Z}\left[\frac{1}{m n}\right] \rtimes \frac{m}{n} \mathbb{Z}$ satisfies $F J C w$, where $m>|n|>0$.

Proof of (1) - (4) can be found for example in [8] section 2.3. (5) is the main result of [1] and [7]. (6) is proved in [8].

\section{Proof of the Main theorem}

In this section, we prove our main theorem. Recall $B S(m, n)=\left\langle a, b \mid b^{-1} a^{m} b=a^{n}\right\rangle$. We assume $m>|n|>1$.

Let $\Gamma(m, n)=\mathbb{Z}\left[\frac{1}{m n}\right] \rtimes \frac{m}{n} \mathbb{Z} . \quad \Gamma(m, n)$ is a solvable linear group. There is a map $\phi$ : $B S(m, n) \rightarrow \Gamma(m, n)$ mapping $a$ to $(1,0)$ and $b$ to $(0,1)$. Note that $\mathrm{FJCw}$ is known for $\Gamma(m, n)$ by Proposition 1.1 (6). Hence by Proposition 1.1 (4) in order to prove FJCw for $B S(m, n)$, we only need to prove $\mathrm{FJCw}$ holds for any subgroup $\phi^{-1}(C)$ of $\Gamma(m, n)$ where $C$ is a cyclic subgroup of $\Gamma(m, n)$.

We proceed to analyze $\phi^{-1}(C)$ using Bass-Serre theory. Part of the idea here is from the proof of Lemma 4.3 in [4].

Viewing $B S(m, n)$ as an HNN extension, we have an associated oriented tree $T(m, n)$ and $B S(m, n)$ acts on it without inversion, see for example [3], I.3.4. Let $H$ be the cyclic 
subgroup in $B S(m, n)$ generated by $a, N$ be the subgroup generated by $a^{m}$. Then the vertices of $T(m, n)$ are left cosets $g H$, and edges are the left cosets $g N$ where $g \in B S(m, n)$. The edge $g N$ connects from the tail vertex $g H$ to the head vertex $g b H$. The stabilizer of the vertex $g \mathrm{H}$ is the subgroup $\mathrm{gHg}^{-1}$, and the stabilizer of the edge $g \mathrm{~N}$ is $\mathrm{gNg}^{-1}$. At each vertex, there are $m$ edges going out and $n$ edges going in. See Figure 1 for a picture of $T(2,3)$.

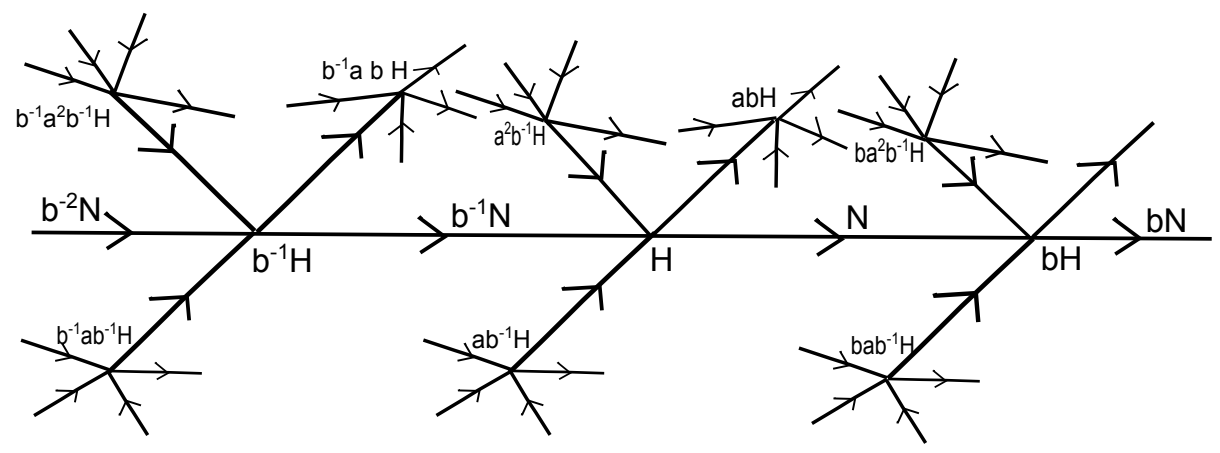

FIGURE 1. $T(2,3)$

Proposition 2.1. If $C$ is generated by $(x, y)$ with $y \neq 0$, then $\phi^{-1}(C)$ is a free group. Hence $\phi^{-1}(C)$ satisfies the FJCw.

Proof We will show $\phi^{-1}(C)$ is a free group by showing it acts freely on the tree $T(m, n)$ (see for example [3], I.4.1). For every vertex $g H$ in $T(m, n)$, its stabilizer under the action of $B S(m, n)$ is the cyclic subgroup $g H g^{-1}$. Let $\phi(g)=(u, v)$, then for any $k \in \mathbb{Z}$,

$$
\phi\left(g a^{k} g^{-1}\right)=\phi(g) \phi\left(a^{k}\right) \phi\left(g^{-1}\right)=(u, v)(k, 0)\left(-\left(\frac{n}{m}\right)^{v} u,-v\right)=\left(\left(\frac{m}{n}\right)^{v} k, 0\right)
$$

Note that $C$ is generated by $(x, y)$ with $y \neq 0$ and the second coordinate of $(x, y)^{n}$ is $n y$. Hence $\phi\left(g \mathrm{Hg}^{-1}\right) \cap C=\{(0,0)\}$. Note that $\phi$ restricted to $H$ is injective, hence it is injective when restricted to $\mathrm{gHg}^{-1}$. Therefore $g \mathrm{Hg}^{-1} \cap \phi^{-1}(C)=\{1\}$. We conclude that for every vertex $g H$, the stabilizer for the action of $\phi^{-1}(C)$ on $T(m, n)$ is trivial. Therefore $\phi^{-1}(C)$ acts freely on $T(m, n)$. Now by Proposition $1.1(5), \phi^{-1}(C)$ satisfies the FJCw.

Proposition 2.2. Let $B=\mathbb{Z}\left[\frac{1}{m n}\right] \rtimes_{\frac{m}{n}}\{0\}$, then $\phi^{-1}(B)$ is a direct limit of $C A T(0)$ groups. Hence it satisfies FJCw.

Corollary 2.3. If $C$ is generated by $(x, 0)$, then $\phi^{-1}(C)$ is a subgroup of $\phi^{-1}(B)$. By Proposition $1.1(1), \phi^{-1}(C)$ also satisfies FJCw.

We now start the proof of Proposition 2.2. By equation (1) in the proof of Proposition 2.1, one sees that $\phi\left(\mathrm{gHg}^{-1}\right) \subset B$ for any $g$. Hence $\phi\left(g \mathrm{Hg}^{-1}\right) \cap B=\phi\left(g \mathrm{Hg}^{-1}\right)$, and $\mathrm{gHg}^{-1} \cap$ 
$\phi^{-1}(B)=g \mathrm{Hg}^{-1}$. Hence vertex or edge stabilizers for the action of $\phi^{-1}(B)$ on $T(m, n)$ are the same as for the $B S(m, n)$ action. In particular, all the vertex and edge stabilizers are isomorphic to $\mathbb{Z}$. Moreover, for every edge $g N$, the stabilizer $G_{g N} \cong \mathbb{Z}$ embeds into the tail vertex stabilizer $G_{g H} \cong \mathbb{Z}$ by multiplying $m$. Correspondingly, $G_{g N} \cong \mathbb{Z}$ embeds into the head vertex stabilizer $G_{g b H} \cong \mathbb{Z}$ by multiplying $n$.

Note if we add one relation $a=1$ to the group $B S(m, n)$ then the new group is isomorphic to $\mathbb{Z}$. Hence there is a surjective homomorphism $f: B S(m, n) \rightarrow \mathbb{Z}$. If we denote the projection from $\mathbb{Z}\left[\frac{1}{m n}\right] \rtimes \frac{m}{n} \mathbb{Z}$ to $\mathbb{Z}$ by $p$, then $f=p \circ \phi$. And if $\beta \in \phi^{-1}(B)$, then $f(\beta)=0$. The action of $\beta$ on $T(m, n)$ has the following key property.

Lemma 2.4. For any $\beta \in \phi^{-1}(B)$, the oriented geodesic connecting $g H$ and $\beta g H$ contains an even number of edges, half of them are compatible with the orientation on $T(m, n)$, while the other half are oppositely oriented.

Proof We first define an "algebraic distance" function $D: T(m, n) \times T(m, n) \rightarrow \mathbb{R}$. Note the tree $T(m, n)$ has a standard metric with edge length 1 . For any two points $P, Q \in$ $T(m, n)$, there is a unique oriented geodesic connecting $P$ to $Q . D(P, Q)$ is the distance from $P$ to $Q$ counted with signs. In more detail, when the geodesic coincide with tree orientation, it contributes positively; otherwise negatively. For example, in Figure $1, D\left(H, b^{n} H\right)=n$, $D\left(H, b a^{2} b^{-1} H\right)=0$. Note $D(P, Q)=-D(Q, P), D(P, Q)+D(Q, R)=D(P, R)$ for any $P, Q, R \in T(m, n)$.

Since $B S(m, n)$ acts on $T(m, n)$ via orientation preserving isometries, $D(g P, g Q)=$ $D(P, Q)$, for any $g \in B S(m, n)$. In particular, $D(H, g H)=D(a H, a g H)=D(H, a g H)$ since $a$ is in the stabilizer of the vertex $H$. And $D(H, g H)=D(b H, b g H)$, hence $D(H, b g H)=$ $D(H, b H)+D(b H, b g H)=1+D(H, g H)$. Note for any $\beta \in \phi^{-1}(B), f(\beta)=0$. Without loss of generality, we can write $\beta$ as a word $a^{s_{1}} b^{t_{1}} a^{s_{2}} b^{t_{2}} \ldots a^{s_{h}} b^{t_{h}}, s_{i}, t_{j} \in \mathbb{Z}$. Then $t_{1}+t_{2}+\cdots t_{h}=0$. Therefore,

$$
\begin{aligned}
D(H, \beta H)=D(H, & \left.a^{s_{1}} b^{t_{1}} a^{s_{2}} b^{t_{2}} \cdots a^{s_{h}} b^{t_{h}} H\right) \\
& =D\left(H, b^{t_{1}} a^{s_{2}} b^{t_{2}} \cdots a^{s_{h}} b^{t_{h}} H\right) \\
& =t_{1}+D\left(H, a^{s_{2}} b^{t_{2}} \cdots a^{s_{h}} b^{t_{h}} H\right) \\
& \cdots \\
& =t_{1}+t_{2}+\cdots t_{h} \\
& =0
\end{aligned}
$$

In general, $D(g H, \beta g H)=D\left(H, g^{-1} \beta g H\right)=0$ since $g^{-1} \beta g \in \phi^{-1}(B)$. The lemma now follows.

Addendum 2.5. Lemma 2.4 is true for any oriented path $\alpha$ connecting $g H$ and $\beta g H$. 
To see this just observe that $\alpha$ can be reduced to the oriented geodesic connecting $g H$ and $\beta g H$ in a finite number of simple moves of the form

$$
\alpha=\alpha_{1} e(-e) \alpha_{2} \longmapsto \alpha_{1} \alpha_{2}
$$

where $e$ is an oriented edge and $-e$ is the same edge with the opposite orientation. (And $\alpha_{1} \alpha_{2}$, ets. means concatenation of paths.)

Summarize what we have so far, the quotient $T(m, n) / \phi^{-1}(B)$ is an oriented graph of groups with the following properties: every vertex or edge stabilizer is isomorphic to $\mathbb{Z}$, the edge stabilizer embeds into its tail vertex stabilizer by multiplying $m$, and to its head vertex stabilizer by multiplying $n$; moreover, every oriented loop $\alpha$ in $T(m, n) / \phi^{-1}(B)$ has an even number of edges, where exactly half of them coincide with the orientation. This is a consequence of Addendum 2.5 and the observation that $\alpha$ lifts to an oriented path $\bar{\alpha}$ in $T(m, n)$ with initial point and end point identified by the action of some element in $\phi^{-1}(B)$.

We will prove that any compact connected subgraph of groups of $T(m, n) / \phi^{-1}(B)$ is $\mathrm{CAT}(0)$, hence $\phi^{-1}(B)$ is a direct limit of $\mathrm{CAT}(0)$ groups which satisfies the $\mathrm{FJCw}$ by Proposition 1.1 (3), (5).

Now let $Y$ be a compact connected subgraph of groups of $T(m, n) / \phi^{-1}(B)$, and $\Gamma$ be its corresponding group. We will construct a $K(\Gamma, 1)$ space with universal cover $\operatorname{CAT}(0)$.

Choosing a base vertex $v_{0}$ in $Y$, since its stabilizer is $\mathbb{Z}$, we construct a cylinder $S^{1} \times[0,1]$ with radius 1 . For every edge and every other vertex, we will construct a cylinder $S^{1} \times[0,1]$ with radius determined inductively by the following criteria:

The radius of the cylinder corresponding to an edge is $m$ times the radius of its tail vertex cylinder, and $n$ times the radius of its head vertex cylinder.

We now explicitly define these radius so that the above criteria are satisfied. We start by defining the radius $r(v)$ of the cylinder corresponding to a vertex $v$ of $Y$ as follows. Let $\alpha$ be an oriented path connecting $v_{0}$ to $v$. Define the integer \#(v) to be the number of positive (agreeing) edges in $\alpha$ minus the number of negative (disagreeing) edges. To see that \#(v) is well defined, let $\gamma$ be a second such path. Then the concatenation $\alpha(-\gamma)$ is an oriented loop in $T(m, n) / \phi^{-1}(B)$ which (as observed above) must have the same number of + edges as - edges. Now let

$$
r(v)=\left(\frac{m}{n}\right)^{(\#(v))}
$$

and then the radius $r(e)$ of an edge $e$ is defined by

$$
r(e)=n\left(\frac{m}{n}\right)^{(\#(v))}
$$

where $v$ is the head vertex of $e$. 
Now we glue all these cylinders together by local isometries according to the graph $Y$. We glue them together using the following method.

Let $S^{1} \times[0,1]$ be a cylinder corresponding to an edge, denote its tail vertex cylinder by $S_{t}^{1} \times[0,1]$ and its head vertex cylinder by $S_{h}^{1} \times[0,1]$. We glue $S^{1} \times\{0\}$ to $S_{t}^{1} \times\{1\}$ by a $m$-fold locally isometric covering projection (orientation preserving if $m>0$ orientation reversing if $m<0$ ). And we glue $S^{1} \times\{1\}$ to $S_{h}^{1} \times\{0\}$ by a $n$-fold locally isometric covering projection (orientation preserving if $n>0$ orientation reversing if $n<0$ ).

Therefore we have constructed a 2-complex which is a model for $K(\Gamma, 1)$. It is now easy to check that its universal cover is a $\operatorname{CAT}(0)$ space; cf. [2], p502, Theorem I.2.7. This completes the proof of Proposition 2.2.

Combining Proposition 2.1 and Corollary 2.3, we prove $\mathrm{FJCw}$ for $B S(m, n)$ by using Proposition 1.1 (4).

\section{REFERENCES}

1. A. Bartels and W. Lück, The Borel Conjecture for hyperbolic and CAT(0)-groups, Annals of Math. 175., pp. 631-689, 2012.

2. W. W. Davis, The geometry and topology of Coxeter groups, London mathematical Society monographs, Vol. 32, 2008.

3. W. Dicks and M. J. Dunwoody, Groups acting on graphs, Cambridge University press, 1989.

4. F. T. Farrell and P. A. Linnell, K-theory of solvable groups. Proc. London Math. Soc. (3), 87(2) : 309-336, 2003.

5. G. Gandini, S. Meinert, H. Rüping, The Farrell-Jones Conjecture for fundamental groups of graphs of abelian groups, arXiv:1309.6909, 2013.

6. F. T. Farrell, X. Wu, Farrell-Jones Conjecture for the solvable Baumslag-Solitar groups, arXiv:1304.4779.

7. C. Wegner, The K-theoretic Farrell-Jones conjecture for CAT(0)-groups, Proc. Amer. Math. Soc. 140 (2012), 779-793.

8. C. Wegner, The Farrell-Jones conjecture for virtually solvable groups, arXiv:1308.2432.

Department of Mathematics, State University of New York at Binghamton, NY, 13902, U.S.A.

E-mail address: farrell@math. binghamton.edu

Department of Mathematics, State University of New York at Binghamton, NY, 13902, U.S.A.

E-mail address: xwu@math.binghamton.edu 